\title{
UNIVERSITY OF MIAMI RADIOCARBON DATES V
}

K L ELDRIDGE, J J STIPP, and J HATTNER

Department of Geology, University of Miami, Coral Gables, Florida

The following list of dates are selected from geologic and archaeologic samples measured in early 1975. The technique employed is liquid scintillation counting of wholly synthesized benzene as described by Noakes et al (1965) and discussed in R, v 16, p 402-408. Errors are reported as one standard deviation.

\section{ACKNOWLEDGMENTS}

We are grateful to D Evans, Dept Biology, for the supplemental use of his Packard Tri-Carb 2003 liquid scintillation spectrometer. We also wish to thank students, P Crevello, S Locker, and J Plescia, for their assistance with various aspects of laboratory preparations.

\section{SAMPLE DESCRIPTIONS}

\section{ARCHAEOLOGIC SAMPLES}

\section{Santa Leticia series}

\section{A. El Salvador}

Three charcoal samples from artificial fill under 'Pot Belly' statue, 2nd terrace, Finca Santa Leticia, Apaneca area, El Salvador (13 $51^{\prime} 18^{\prime \prime}$ N, 89 $47^{\circ} 32^{\prime \prime}$ W). Coll 1969 and subm 1974 by S H Boggs.

General Comment (SHB): results indicate emplacement of statuary during Pre-Classic era. Culture presently unknown. Santa Leticia statuary emplacement corresponds chronologically with 'Pot Belly' emplacements at Finca Monte Alto, Guatemala (Berger, 1973; Cadwell et al, 1975).

UM-390. Santa Leticia 1

UM-391. Santa Leticia 3

\section{UM-392. Santa Leticia 19-21}

\section{B. Puerto Rico}

\section{Villa Taina series}

Charcoal and shell from shell midden, Boquerón, Puerto Rico $\left(18^{\circ} 02^{\prime} 27^{\prime \prime} \mathrm{N}, 67^{\circ} 11^{\prime} 33^{\prime \prime} \mathrm{W}\right)$. Charcoal pretreated with $5 \% \mathrm{NaOH}$ for removal of humic acid. Dated to establish Arawak Indian habitation (Goodwin, 1973). Coll and subm 1974 by C Goodwin.

UM-398. Villa Taina 1Aa

$1300 \pm 90$

Charcoal from $27 \mathrm{~cm}$ beneath surface.
$2400 \pm 60$

450 вС

$$
2460 \pm 130
$$$$
510 \text { BC }
$$

$2780 \pm 210$

830 BC AD 650 
UM-399. Villa Taina 1Ab

Duplicate run of UM-398.

\section{UM-400. Villa Taina 1B}

Shell from $30 \mathrm{~cm}$ beneath surface.

II. GEOLOGIC SAMPLES

\section{A. United States}

\section{Caesars Creek Bank series}

Shell and coral samples from 8 piston cores from .2 to $1.7 \mathrm{~m}$ water, Caesars Creek Bank, Biscayne Bay, Florida. Carbonate mudbank—storm, tidal delta assoc with major tidal pass between Biscayne Bay and inner reef tract, SE coast of Florida. Dates depositional sequence of bank. Samples found in situ except UM-336 and -344. Coll and subm 1974 by E R Warzeski, RSMAS, Univ Miami.

Core 1 from $.2 \mathrm{~m}$ water. Core penetration $4.8 \mathrm{~m}$ to bedrock $\left(25^{\circ} 23^{\prime} 00^{\prime \prime} \mathrm{N}\right.$, $\left.80^{\circ} 13^{\prime} 12^{\prime \prime} \mathrm{W}\right)$.

\section{UM-297. Core 1}

$1760 \pm 100$

Codakia orbicularis and Porites divaricata from 135 to $145 \mathrm{~cm}$ within core.

\section{UM-326. Core 1}

Codakia orbicularis from 290 to $300 \mathrm{~cm}$ within core.

\section{UM-327. Core 1}

$1350 \mathrm{BC}$

Codakia orbicularis from 335 to $345 \mathrm{~cm}$ within core.

$$
3870 \pm 80
$$

1920 BC

Core 2 from $.5 \mathrm{~m}$ water. Core penetration $5.3 \mathrm{~m}$ to bedrock $\left(25^{\circ} 22^{\prime} 53^{\prime \prime}\right.$ $\left.\mathrm{N}, 80^{\circ} 13^{\prime} 01^{\prime \prime} \mathrm{W}\right)$.

\section{UM-335. Core 2}

Codakia orbicularis, Astrea tecta americana, and Porites divaricata from 150 to $160 \mathrm{~cm}$ within core.

UM-336. Core 2

$4200 \pm 100$ 2250 вC

Anodontia alba from 420 to $430 \mathrm{~cm}$ within core. Comment (ERW): shell directly underlying storm mud layer. Shell was deposited after burial of UM-337.

UM-337. Core 2

Anodontia alba and Laevicardium laevigatum from 480 to $500 \mathrm{~cm}$ within core. 


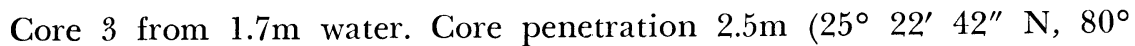
$\left.12^{\prime} 50^{\prime \prime} \mathrm{W}\right)$.

\section{UM-322. Core 3}

Codakia orbicularis from 235 to $245 \mathrm{~cm}$ within core.

Core 4 from $1.7 \mathrm{~m}$ water. Core penetration $4.6 \mathrm{~m}$ to bedrock $\left(25^{\circ} 22^{\prime}\right.$ $\left.41^{\prime \prime} \mathrm{N}, 80^{\circ} 12^{\prime} 48^{\prime \prime} \mathrm{W}\right)$. Comment (ERW): core penetrated buried tidal channel. UM-332 antedates cutting of channel and is below erosional surface of channel floor. UM-331, -344, and -330 record lateral migration of channel margin across core site.

\section{UM-330. Core 4}

$1540 \pm 80$

Porites divaricata from 80 to $90 \mathrm{~cm}$ within core.

UM-344. Core 4

$$
\text { AD } 410
$$

$3530 \pm 130$

$1580 \mathrm{BC}$

Porites divaricata, Astrea tecta americana, and Tellina similis from 255 to $265 \mathrm{~cm}$ within core. Comment (ERW): UM-344 appears to be transported material.

UM-331. Core 4

$1880 \pm 80$

Anodontia alba from $310 \mathrm{~cm}$ within core.

UM-322. Core 4

$3650 \pm 100$

1700 BC

Laevicardium laevigatum from 360 to $370 \mathrm{~cm}$ within core.


$\left.12^{\prime \prime} \mathrm{W}\right)$.

\section{UM-321. Core 5}

Laevicardium laevigatum from 370 to $410 \mathrm{~cm}$ within core.

UM-320. Core 5

$3480 \pm 90$

$1520 \mathrm{BC}$

Laevicardium laevigatum from 480 to $510 \mathrm{~cm}$ within core.

Core 6 from $.4 \mathrm{~m}$ water. Core penetration $5.4 \mathrm{~m}\left(25^{\circ} 22^{\prime} 58^{\prime \prime} \mathrm{N}, 80^{\circ} 12^{\prime}\right.$ $\left.15^{\prime \prime} \mathrm{W}\right)$.

UM-318. Core 6

$\mathbf{2 0 2 0} \pm 90$

$70 \mathrm{BC}$

Laevicardium laevigatum and Porites divaricata from 360 to $380 \mathrm{~cm}$ within core.

UM-319. Core 6

Laevicardium laevigatum from 460 to $480 \mathrm{~cm}$ within core. 
Core 7 from $.4 \mathrm{~m}$ water. Core penetration $5.4 \mathrm{~m}$ to bedrock $\left(25^{\circ} 22^{\prime} 21^{\prime \prime}\right.$ N, $\left.80^{\circ} 12^{\prime} 48^{\prime \prime} \mathrm{W}\right)$.

UM-323. Core 7

$990 \pm 80$

Manicina areolata from 280 to $290 \mathrm{~cm}$ within core.

UM-324. Core 7

$3530 \pm 130$

1580 BC

Laevicardium laevigatum, Tellina mera, and Cumingia tellinoides from 485 to $495 \mathrm{~cm}$ within core.

Core 8 from $.9 \mathrm{~m}$ water. Core penetration $3.9 \mathrm{~m}\left(25^{\circ} 22^{\prime} 16^{\prime \prime} \mathrm{N}, 80^{\circ} 13^{\prime}\right.$ $\left.00^{\prime \prime} \mathrm{W}\right)$.

UM-333. Core 8

$\mathbf{2 4 0} \pm 80$

Astrea tecta americana, Natica canrena, and Porites divaricata from 200 to $210 \mathrm{~cm}$ within core.

UM-334. Core 8

$1920 \pm 120$

Manicina areolata from 310 to $330 \mathrm{~cm}$ within core.

AD 30

\section{Safety Valve series}

Eight cores from Safety Valve tidal bar, Biscayne Bay, Florida. Dates establish pattern of tidal-bar formation relative to sea level rise (Plescia et al, 1975). Cores from .5 to $1 \mathrm{~m}$ water. Core A $\left(25^{\circ} 39^{\prime} 03^{\prime \prime} \mathrm{N}\right.$, $\left.80^{\circ} 10^{\prime} 25^{\prime \prime} \mathrm{W}\right)$. Core B $\left(25^{\circ} 39^{\prime} 06^{\prime \prime} \mathrm{N}, 80^{\circ} 10^{\prime} 05^{\prime \prime} \mathrm{W}\right)$. Core C $\left(25^{\circ}\right.$ $\left.37^{\prime} 44^{\prime \prime} \mathrm{N}, 80^{\circ} 10^{\prime} 13^{\prime \prime} \mathrm{W}\right)$. Core D (25 $\left.37^{\prime} 48^{\prime \prime} \mathrm{N}, 80^{\circ} 10^{\prime} 00^{\prime \prime} \mathrm{W}\right)$. Core E $\left(25^{\circ} 37^{\prime} 12^{\prime \prime} \mathrm{N}, 80^{\circ} 10^{\prime} 05^{\prime \prime} \mathrm{W}\right)$. Core F (25 $\left.36^{\prime} 25^{\prime \prime} \mathrm{N}, 80^{\circ} 10^{\prime} 30^{\prime \prime} \mathrm{W}\right)$. Core G $\left(25^{\circ} 36^{\prime} 00^{\prime \prime} \mathrm{N}, 80^{\circ} 10^{\prime} 00^{\prime \prime} \mathrm{W}\right)$. Core $\mathrm{H}\left(25^{\circ} 36^{\prime} 16^{\prime \prime} \mathrm{N}, 80^{\circ} 09^{\prime}\right.$ $\left.45^{\prime \prime} \mathrm{W}\right)$. Coll and subm 1973, 1974 by J Plescia, Univ Miami.

UM-309. Core A

Shell from 12 to $18 \mathrm{~cm}$ within core.

UM-505. Core A

Shell from 48 to $56 \mathrm{~cm}$ within core.

UM-306. Core A

Porites coral from 61 to $69 \mathrm{~cm}$ within core.

UM-495. Core A

Porites coral from 152 to $158 \mathrm{~cm}$ within core.

UM-308. Core A

Shell from 335 to $363 \mathrm{~cm}$ within core.
$900 \pm 60$

$1700 \pm 80$ AD 250

$1500 \pm 80$ AD 450

$$
1520 \pm 120
$$
AD 430

$3020 \pm 110$ 1070 BC 
UM-307. Core B

Shell from 399 to $424 \mathrm{~cm}$ within core.

UM-310. Core B

Recrystallized limestone from 424 to $427 \mathrm{~cm}$ within core.

UM-516. Core C

Shell from 175 to $182 \mathrm{~cm}$ within core.

UM-514. Core C

Shell from 250 to $262 \mathrm{~cm}$ within core.

UM-515. Core D

Shell from 71 to $79 \mathrm{~cm}$ within core.

UM-517. Core D

Porites coral from 71 to $79 \mathrm{~cm}$ within core.

UM-513. Core D

Shell from 320 to $343 \mathrm{~cm}$ within core.

UM-502. Core E

Porites coral from 0 to $10 \mathrm{~cm}$ within core.

UM-499. Core E

Porites coral from 24 to $32 \mathrm{~cm}$ within core.

UM-496. Core E

Shell from 52 to $55 \mathrm{~cm}$ within core.

UM-498. Core $\mathbf{E}$

Shell from 175 to $183 \mathrm{~cm}$ within core.

UM-503. Core E

Shell from 183 to $193 \mathrm{~cm}$ within core.

UM-511. Core $\mathbf{F}$

Porites coral from 14 to $22 \mathrm{~cm}$ within core.

UM-510. Core F

Porites coral from 57 to $67 \mathrm{~cm}$ within core.

$$
960 \pm 70
$$$$
\text { AD } 990
$$

$$
2360 \pm 90
$$

$410 \mathrm{BC}$

$$
1380 \pm 70
$$
AD 570

$1230 \pm 80$ AD 720

$2500 \pm 120$

550 BC

AD 1110

$840 \pm 80$ AD 1430

$520 \pm 60$

$960 \pm 70$ AD 990

$4030 \pm 110$ 2080 BC

$3130 \pm 110$

$1180 \mathrm{BC}$

$320 \pm 70$

AD 1630

$660 \pm 70$

AD 1290 


\section{UM-509. Core F}

Porites coral from 159 to $168 \mathrm{~cm}$ within core.

UM-507. Core F

Porites coral from 210 to $216 \mathrm{~cm}$ within core.

UM-508. Core F

Shell from 259 to $269 \mathrm{~cm}$ within core.

UM-512. Core F

Recrystallized limestone from 259 to $269 \mathrm{~cm}$ within core.

UM-506. Core G

Porites coral from 261 to $270 \mathrm{~cm}$ within core.

UM-500. Core $\mathbf{H}$

Porites coral from 25 to $33 \mathrm{~cm}$ within core.

\section{UM-497. Core H}

Porites coral from 46 to $53 \mathrm{~cm}$ within core.

\section{UM-504. Core $\mathbf{H}$}

Porites coral from 86 to $94 \mathrm{~cm}$ within core.

\section{UM-501. Core H}

Porites coral from 195 to $203 \mathrm{~cm}$ within core.

$$
1470 \pm 80
$$$$
\text { AD } 480
$$

$$
2230 \pm 80
$$

280 BC

$4270 \pm 100$

2320 BC

$19,840 \pm 420$ $17,890 \mathrm{BC}$

$$
1900 \pm 140
$$

AD 50

$$
4200 \pm 90
$$

2250 вC

$$
\text { AD } 1430
$$

$$
520 \pm 80
$$

AD 1050

$$
900 \pm 80
$$

$900 \pm 80$

AD 1050

\section{B. Bahamas}

\section{Frazers Hog Cay series}

Carbonate sediment from 3 cores, Frazers Hog Cay, Bahamas. Continuation of study on Frazers Hog Cay (R, v 17, p 410), to determine date of Holocene bank flooding and transgression (Crevello et al, 1975). Coll 1962 by J Imbrie; subm 1975 by P Crevello, Univ Miami, and H Buchanan.

UM-488. Core 855 (ce) R

$$
2240 \pm 60
$$

$$
290 \text { вC }
$$

Oolitic sand from 242 to $262 \mathrm{~cm}$ within core. From $.75 \mathrm{~m}$ water $\left(25^{\circ} 26^{\prime} 56^{\prime \prime} \mathrm{N}, 77^{\circ} 56^{\prime} 45^{\prime \prime} \mathrm{W}\right)$.

\section{UM-489. Core 858N}

$$
2120 \pm 70
$$

Shells from $120 \mathrm{~cm}$ within core. From $2.2 \mathrm{~m}$ water $\left(25^{\circ} 27^{\prime} 25^{\prime \prime} \mathrm{N}\right.$, $77^{\circ} 53^{\prime} 14^{\prime \prime} \mathrm{W}$ ). 
UM-490. Core 784-2 BN

$960 \pm 60$

Organic aggregate and grapestone sand from 20 to $30 \mathrm{~cm}$ within core. From $1.5 \mathrm{~m}$ water $\left(25^{\circ} 27^{\prime} 25^{\prime \prime} \mathrm{N}, 77^{\circ} 53^{\prime} 14^{\prime \prime} \mathrm{W}\right)$.

\section{UM-491. Core 784-2 BN}

$1400 \pm 100$

Organic aggregate and grapestone sand from $150 \mathrm{~cm}$ within core. Same core as UM-490.

\section{Haines Cay series}

Marine-derived carbonates from beach and eolian dune ridge sediments. Dates provide temporal framework for interpretation of island fomation and Holocene sea level. Continuation of study on Haines Cay, Bahamas (R, v 17, p 118; Pasley et al, 1975). Dune A forms rocky shoreline on NE side of I. Lithified material is well-sorted, oolitic calcarenite. Dune B is W of Dune A. Semi-lithified material is oolitic, pelletoidal calcarenite. Dune C forms shoreline on NW side of I. Lithified material is well-sorted, oolitic calcarenite. Dune D is a massive back beach dune $\mathrm{S}$ of Dune A. Poorly lithified material is oolitic, pelletoidal calcarenite. Dune E extends S of Dunes A and B, W of Dune D. Semilithified material is oolitic, pelletoidal calcarenite. Coll and subm 1974 by D Pasley, RSMAS, Univ Miami, and S Locker.

UM-407. Dune A

$5580 \pm 100$

Fine grained oolites, alt $2 \mathrm{~m}$ above MSL $\left(25^{\circ} 44^{\prime} 10^{\prime \prime} \mathrm{N}, 77^{\circ} 49^{\prime}\right.$ $\left.08^{\prime \prime} W\right)$.

\section{UM-494. Dune A}

$6280 \pm 100$

4330 BC $\left.07^{\prime \prime} \mathrm{W}\right)$

Fine grained oolites, alt $2 \mathrm{~m}$ above MSL $\left(25^{\circ} 34^{\prime} 58^{\prime \prime} \mathrm{N}, 77^{\circ} 49^{\prime}\right.$

\section{UM-404. Dune A}

$5840 \pm 100$

3890 вC

Fine grained oolites, alt $1.5 \mathrm{~m}$ above MSL $\left(25^{\circ} 43^{\prime} 58^{\prime \prime} \mathrm{N}, 77^{\circ} 49^{\prime}\right.$ 07" W). Comment (SL): UM-494 and -404 show reverse age trend relative to superposition.

\section{UM-409. Dune B}

$4110 \pm 111$

Medium grained oolites, alt $7 \mathrm{~m}$ above MSL $\left(25^{\circ} 44^{\prime} 10^{\prime \prime} \mathrm{N}, 77^{\circ} 49^{\prime}\right.$ $\left.08^{\prime \prime} \mathrm{W}\right)$.

$2160 \mathrm{BC}$

\section{UM-408. Dune B}

$3670 \pm 90$

Medium grained oolites, alt $5 \mathrm{~m}$ above MSL $\left(25^{\circ} 44^{\prime} 10^{\prime \prime} \mathrm{N}, 77^{\circ}\right.$ $\left.49^{\prime} 08^{\prime \prime} \mathrm{W}\right)$. Comment (SL): UM-409 and -408 show reverse age trend relative to superposition. 


\section{UM-492. Dune $C$}

Fine grained oolites, alt $\mathrm{lm}$ above MSL $\left(25^{\circ} 44^{\prime} 09^{\prime \prime} \mathrm{N}, 77^{\circ} 49^{\prime}\right.$ $\left.12^{\prime \prime} \mathrm{W}\right)$.

4510 BC

\section{UM-405. Dune D}

$1920 \pm 80$

Medium grained oolites, alt $2 \mathrm{~m}$ above MSL (25 $43^{\prime} 58^{\prime \prime} \mathrm{N}, 77^{\circ} 49^{\prime}$ $\left.07^{\prime \prime} \mathrm{W}\right)$.

\section{UM-493. Dune E}

Medium grained oolites, alt $2 \mathrm{~m}$ above MSL $\left(25^{\circ} 43^{\prime} 54^{\prime \prime} \mathrm{N}, 77^{\circ} 49^{\prime}\right.$ $\left.09^{\prime \prime} \mathrm{W}\right)$.

\section{UM-406. Beach sand}

$1860 \pm 70$

Mixed carbonate sand from intertidal zone, $\mathrm{E}$ of Dunes $\mathrm{D}$ and $\mathrm{E}$ $\left(25^{\circ} 43^{\prime} 57^{\prime \prime} \mathrm{N}, 77^{\circ} 49^{\prime} 07^{\prime \prime} \mathrm{W}\right)$.

\section{Martinique series}

\section{Martinique}

Charcoal from pyroclastic surge sediments, near Mt Pelée, Martinique. Dated to determine age of surge sediments eminating from $\mathrm{Mt}$ Pelée. Coll and subm 1974 by G P L Walker, Imperial Coll Sci and Technol, London.

\section{UM-394. Martinique 56}

$1230 \pm 80$

Sample from non-pumiceous sediment, $2.6 \mathrm{~km} \mathrm{~S}$ of Mt Pelée summit $\left(14^{\circ} 47^{\prime} \mathrm{N}, 61^{\circ} 10^{\prime} \mathrm{W}\right)$.

\section{UM-395. Martinique 75}

$3110 \pm 100$

Sample from non-pumiceous sediment, .5km NW of Quartière Démare, NE slope of Mt Pelée $\left(14^{\circ} 50^{\prime} \mathrm{N}, 61^{\circ} 07^{\prime} \mathrm{W}\right)$. Comment (GPLW): precedes UM-396 surge sediment.

\section{UM-396. Martinique 95}

$2020 \pm 80$

Sample from pumice sediment, road cut at Morne Calebasse, $2.6 \mathrm{~km}$ SE of Mt Pelée summit $\left(14^{\circ} 48^{\prime} \mathrm{N}, 61^{\circ} 09^{\prime} \mathrm{W}\right)$.

\section{UM-397. Martinique 131}

$4940 \pm 100$

Sample from non-pumiceous sediment, road cut $.4 \mathrm{~km}$ SW of Morne Rouge $\left(14^{\circ} 46^{\prime} \mathrm{N}, 61^{\circ} 08^{\prime} \mathrm{W}\right)$. 


\section{RFFERFNCFS}

Berger, R, 1973, Summary of UCLA ${ }^{14} \mathrm{C}$ dates for Monte Alto.

Cadwell, H, Boggs, S H, and Stipp, J J, 1975, Radiocarbon dating of the Pot-Belly statuary of El Salvador and Guatemala: Florida Acad Sci, Lakeland, Florida, March 20-22.

Crevello, P, Buchanan, H, and Stipp, J J, 1975, Radiocarbon dates and recent Bahamian sea level: Florida Acad Sci, Lakeland, Florida, March 20-22.

Eldridge, K L, Stipp, J J, Hattner, J, and McDougal, E, 1975, University of Miami radiocarbon dates IV: Radiocarbon, v 17, p 407-412.

Goodwin, C, 1973, Villa Taina: 5th internatl cong for the study of Pre-Columbian cultures of the Lesser Antilles Proc, p 1-10.

Noakes, J E, Kim, S M, and Stipp, J J, 1965, Chemical and counting advances in liquid scintillation age dating: 6th internatl ${ }^{14} \mathrm{C}$ and ${ }^{3} \mathrm{H}$ dating conf Proc, Pullman, Washington, June 7-11, 1965, p 68-92.

Pasley, D, Locker, S, Stipp, J J, 1975, Holocene sea level history and its relationship to eolianite sequences on Haines Cay, Berry Islands, Bahamas: Florida Acad Sci, Lakeland, Florida, March 20-22.

plescia, J B, Stipp, J J, 1975, Preliminary geochronology of the safety valve formation: Florida Acad Sci, Lakeland, Florida, March 20-22.

Stipp, J J, and Eldridge, K L, 1975, University of Miami radiocarbon dates II: Radiocarbon, v 17, p 112-120.

Stipp, J J, Eldridge, K L, Cohen, S J, and Webber, K, 1974, University of Miami radiocarbon dates I: Radiocarbon, v 16, p 402-408. 